\title{
An Application of High-Resolution Dual-Lens Dark-Field Electron Holography in Strain Analysis for Nanometer Semiconductor Device in Wafer-foundries
}

\author{
Wayne Zhao*, Yun-yu Wang**, and Bianzhu Fu* \\ Physical Failure Analysis, Center for Complex Analysis, Characterization Group, \\ Fab8 at Malta*, and Fab10 at Fishkill**, GLOBALFOUNDRIES, New York, USA.
}

Constantly striving for miniaturization of semiconductor device with nanometer transistors as the basic building block is the theme of efforts in wafer-foundries [1 7]. To synchronize with the device shrinkage, novel techniques to enhance carrier mobility within constrained geometry are developed. Several techniques can be realistically applied in the strain-engineering for Si-based nanometer transistors [4 7]. For example, SiGe, as a stressor, is introduced in p-type transistors [4]. Since a nanometer transistor device is basically a nanometer-scale composite, to effectively understand appropriate levels and desired distributions of strains applied into nanometer transistor devices is a big challenge to process integration, device characterizations, and physical failure analysis (PFA) teams.

Dark-field electron holography acquired by a Lorentz lens or a mini-condenser lens has been employed to characterize strain measurement. However, fundamentally there are two intrinsic limitations for the conventional industrial approach. One is the limited fringe spacing (e.g., 5 6nm), which is acceptable to device for $20 \mathrm{~nm}$ or above, especially for a "planar" transistor built on bulk-Si substrate or Si-oninsulator (SOI), but hard to extend to FinFET with a Fin dimension of $14 \mathrm{~nm}$ or below. Another obstacle was extremely time-consuming to tilt to the desired crystal zone under a typical two-beam electron diffraction condition.

Yun-yu Wang's innovations [5 7] overcome these technical barriers. By introducing a dual lens setting with combined contributions of Lorentz lens and objective lens with various suitable strengths in a modern Analytical TEM, Wang's pioneer work not only extends the fringe spacing to a higherresolution (e.g., $0.5 \mathrm{~nm}$ ) range, but also enables large zone-tilt at high objective lens currents as a routine. Collectively, these two innovative settings open a door to extend dark field electron holography for strain measurement at a high spatial resolution into the era of FinFET, 14nm or even below.

One example demonstrated here is an application of Wang's patented method in a strain characterization on an enhancement of device performance by applying SiGe into p-FET device. Key parameters achieved on a Cs image corrected Titan at Fab 8, by the dual lens electron holography aforementioned, include: (1) fringe width: $219 \mathrm{~nm}$; (2) fringe spacing: up to $0.4 \mathrm{~nm}$; (3) fringe contrast: over 20\% ; (4) dark field holography on a 28LPQ test device to obtain strain map of $\langle 220\rangle$ and $\langle 004\rangle$ diffractions; and (5) level of strains, measured at top of $\mathrm{Si}:-1.6 \%$, and $0.8 \%$, for $\langle 220\rangle$ and $\langle 004\rangle$, respectively. Clearly, strains indeed had been applied into the desired p-FET transistor gate channel. Figure-1a summarizes fringe spacing that can be accomplished versus strength of objective lens. Figure-1b displays an empty test hologram in vacuum, with fringe spacing of $0.6 \mathrm{~nm}$ and fringe contrast of $24 \%$ (Fig-1c). Figure-1d is the device with strain in transistor gate analyzed in Figs-2 \& -3 . Figures-2a 2d are the dark-field electron hologram on $\langle 220\rangle$ and $\langle 400\rangle$ diffractions, respectively, on a device with SiGe embedded in Si-substrate. Figures $3 \mathrm{a} \sim 3 \mathrm{~d}$ are corresponding post-processed strain maps and extracted profiles. 


\section{References:}

[1] W. Zhao, et al., Microscopy \& Microanalysis, Vol. 20 (Supplement 3), (2014), pp.362 363.

[2] W. Zhao, et al., Proc. $38^{\text {th }}$ International Symposium for Testing and Failure Analysis, (2012), pp. 347 355.

[3] W. Zhao, Symp. Proc. the Material Research Society, 2002 Fall Meeting, (2002), Vol. 738, pp. G7.15.1 6.

[4] K. J. Kuhn, et al., ECS Transactions, Vol. 33 (6), (2010), pp. 3-17.

[5] Y. Y. Wang, et al., Ultramicroscopy, 101, (2004), pp. $63-72$.

[6] Y. Y. Wang, et al., Ultramicroscopy, 124, (2013), pp. 117 - 129.

[7] Y.Y. Wang, et al., US patent: US 7,015,469 B2 (2006).

[8] Thanks to Fab8 Management and Legal teams for supporting the publication clearance.
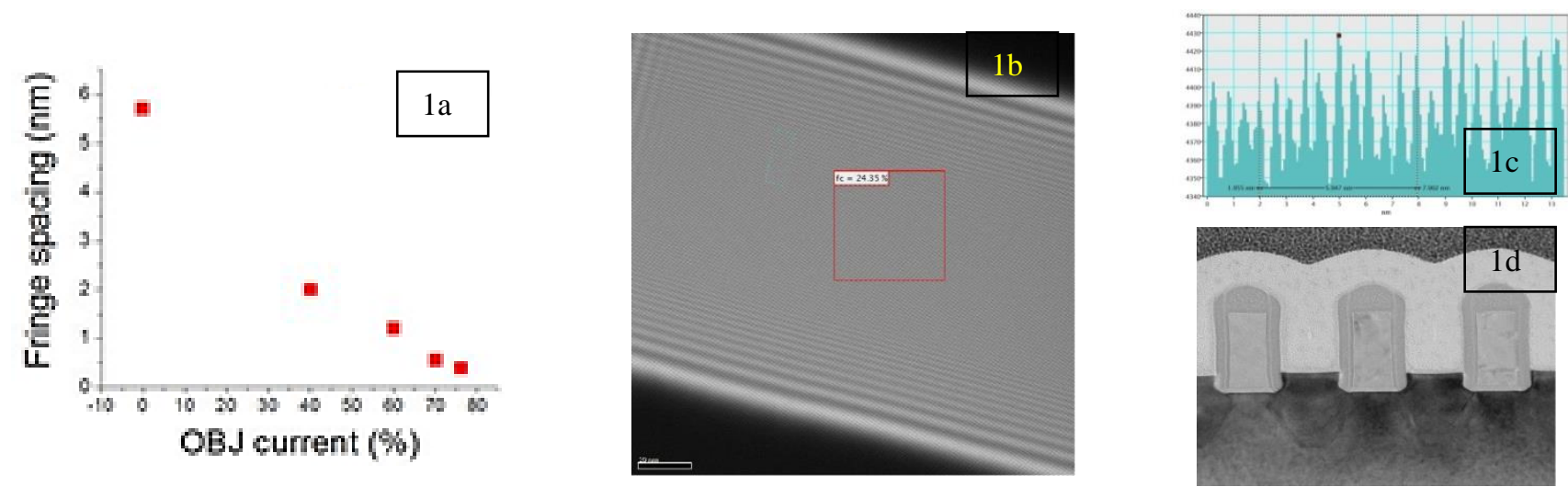

Figure 1. (a) fringe spacing vs. objective lens current; (b) and (c) a high-resolution electron hologram in vacuum, and (d) the device with SiGe stressor, with strains analyzed in Figures-2 and Figures-3.
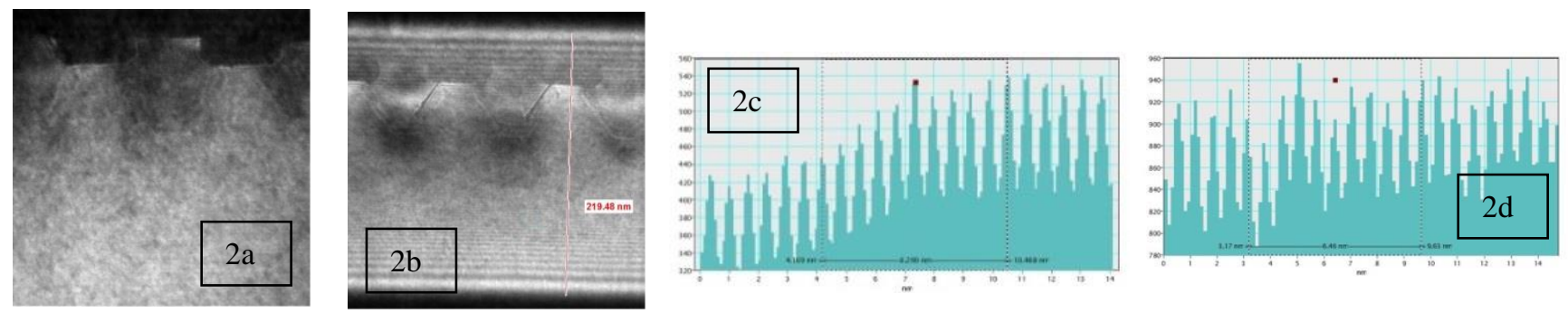

Figure 2. Dark-field electron holograms, (a) and (b), on $\langle 220\rangle$ and $\langle 004\rangle$ diffraction, respectively; and the corresponding proof of high-resolution fringe spacing, (c) and (d); for the device in Figure-1d.
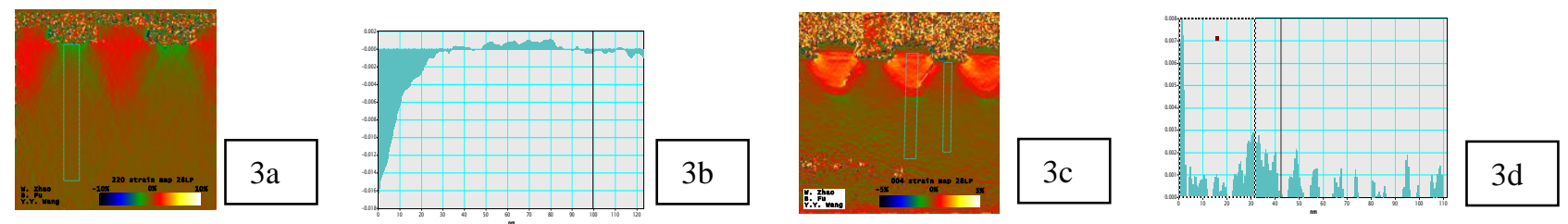

Figure 3. Post-processed strain mapping (a and c); and extracted profiles (b and d), on $\langle 220\rangle$ and $<004>$, respectively. 